\title{
The stellar metallicity distribution of the Galaxy from the BATC survey
}

\author{
Xiyan Peng ${ }^{1,2,3 \star}$, Cuihua $\mathbf{D u}^{3}$, Zhenyu $\mathbf{W} \mathbf{u}^{1,2}$, \\ Jun $\mathbf{M a}^{1,2}$ and $\mathrm{Xu} \mathbf{Z h o u}^{1,2}$ \\ ${ }^{1}$ National Astronomical Observatories, Chinese Academy of Sciences, Beijing 100012, \\ P. R. China \\ ${ }^{2}$ Key Laboratory of Optical Astronomy, National Astronomical Observatories, \\ Chinese Academy of Sciences, Beijing 100012, P. R. China \\ email: xypeng@bao.ac.cn \\ ${ }^{3}$ School of physics, University of the Chinese Academy of Sciences, Beijing 100049, P. R. China \\ email: ducuihua@ucas.ac.cn
}

\begin{abstract}
Using 2200 BATC main sequence (MS) stars which include SDSS stellar atmospheric parameters, we develop the polynomial photometric calibration method to evaluate the stellar effective temperature and metallicity for BATC data. This calibration method has been applied to about $160000 \mathrm{MS}$ stars. The mean stellar metallicity smoothly decreases from -0.65 to $-0.78 \mathrm{dex}$ in the interval $0.5<|Z| \leqslant 2 \mathrm{kpc}$. Metallicity distributions in the halo and the thick disk seem invariant with the distance from the Galactic plane.
\end{abstract}

Keywords. Galaxy: disk - Galaxy: halo- Galaxy: metallicity - Galaxy: formation.

\section{The metallicity distribution}

Based on the stellar atmospheric parameters from SDSS spectra for $\sim 2200$ MS stars which were also observed in the BATC photometric system, we develop the polynomial photometric calibration method to evaluate the stellar effective temperature and metallicity for BATC photometric data. This calibration method has been applied to about $160000 \mathrm{MS}$ stars from 67 BATC observed fields. Color index and magnitude are used to choose the MS stars.

There is a peak in the stellar metallicity distribution at about -1.5 in the interval $5<|Z| \leqslant 14 \mathrm{kpc}$ which corresponds to the halo. The halo metallicity distribution seems invariant with the distance from the plane while the mean thin disk $(0.5<|Z| \leqslant$ $2 \mathrm{kpc}$ ) metallicity smoothly decreases from -0.65 to -0.78 dex. There is a mean vertical metallicity gradient $\mathrm{d}[\mathrm{Fe} / \mathrm{H}] / \mathrm{dz}=-0.1 \mathrm{dex} \mathrm{kpc}^{-1}$ for the thin disk stars. The mean vertical gradient about $-0.03 \pm 0.02 \mathrm{dex} \mathrm{kpc}^{-1}$ for the halo is agreement with the previous work (Du et al. 2006; Peng et al. 2012). In the interval $2<|Z| \leqslant 5 \mathrm{kpc}$, there is a mixture of halo stars and thick disk stars. The peak of the metallicity distribution for the thick disk is about -0.7 dex. Stars with $[\mathrm{Fe} / \mathrm{H}]>-1$ and $2<|Z| \leqslant 5 \mathrm{kpc}$ are selected to determine the metallicity gradient for the thick disk. A vertical metallicity gradient $\sim-0.03 \pm 0.02 \mathrm{dex} \mathrm{kpc}^{-1}$ is found in the thick disk. We believe that the possible cutoff of the thick disk stars is about $5 \mathrm{kpc}$ above and below the Galactic plane which is consistent with the result of the Ivezic et al. (2008). At any distance interval, the variation of the mean metallicity distributions with Galactic longitude is almost flat.

\section{References}

Ivezić Ž., et al., 2008, ApJ, 684, 287

Peng X. Y., Du C. H., Wu Z. Y., 2012, MNRAS, 422, 2756

Du C. H., et al., 2006, MNRAS, 372, 1304 